\title{
FREQUENCY DOMAIN MODELING OF EXTERNAL SYSTEMS IN AN ELECTRO-MAGNETIC TRANSIENTS PROGRAM
}

\author{
A. Semlyen \\ M.R. Iravani \\ Department of Electrical Engineering \\ University of Toronto \\ Toronto, Ontario \\ Canada M5S 1A4
}

\begin{abstract}
The standard EMTP (Electro-Magnetic Transients Program) is based on time domain calculations as these are eminently appropriate for simulation purposes. This paper presents an approach for the direct frequency domain representation of an external system of any size or complexity. The requirements for the external system are that it must be linear and time invariant, and it should include a transmission line connecting it to the study zone and providing a travel delay $\tau$. It is represented in the phase domain and does not have to be symmetrical. At the connecting bus between the study zone and the external system, Fourier transforms provide the conversion from time domain to frequency domain and vice versa. The FFTs are however performed only at time intervals $2 \tau$ so that the approach is computationally efficient especially in the case of large external systems. One important advantage of the method is that the accuracy and simplicity of direct frequency domain modeling is preserved since no approximations or fitting are necessary, even for complex, three-phase frequency dependent components.
\end{abstract}

Keywords: Electromagnetic Transients; Time-Domain Analysis; Frequency-Domain Analysis; Hybrid Simulation.

\section{INTRODUCTION}

The calculation of electro-magnetic transients can be performed by either time or frequency domain methods. The advantage of the forme (the EMTP -- Electro-Magnetic Transients Program [1] -- being the principal program in this group), is due to the direct step by step integration of the ODEs (Ordinary Differential Equations) representing the terminal system components and to the computational decoupling between components separated by transmission lines (because of the travel delay of waves propagating on these lines). Direct time domain simulation also permits the representation of switches and nonlinear elements in a fairly straightforward way. However, basic transmission line modeling is initially always performed in the frequency domain since complex field phenomena in the ground and inside the conductor result in parameters which are functions of frequency. Frequency domain methods have therefore an intrinsic advantage in overcoming the difficulties related to transmission line modeling. Nevertheless, because of its overwhelming computational efficiency, the EMTP has prevailed as a practical tool for power systems applications. It is fundamentally a pure time domain program.

This paper presents a method which permits to link frequency domain component models (such as lines with simple or complex fre quency dependent terminations, and external networks) to the EMTP. Thus a hybrid EMTP is created which preserves all advantages of the standard EMTP while adding the convenience of direct frequency domain modeling of some "peripheral" components. These are linked to the EMTP by means of Fourier transforms.

The basic ideas underlying the linking of Frequency Domain Components (FDC) with the Time Domain Part (TDP) of the EMTP are the following (see Figure 1):

92 WM 304-6 PWRS A paper recommended and approved by the IEEE Power System Engineering Committee of the IEEE Power Engineering Society for presentation at the IEEE/PES 1992 Winter Meeting, New York, New York, January 26 - 30, 1992. Manuscript submitted August 30,1991 ; made available for printing December 31, 1991. (a) The FDC must be linear and time invariant (no inside switching) and must include a transmission line to link it with the the TDP where the electro-magnetic transient phenomenon is initiated. This line is necessary in order to provide the time delay $\tau$ (the ideal travel delay along the line) needed for the computations, as explained next.

(b) The principle of causality assures that an input $x$ at $A$ (the reflected wave from TDP) to the FDC line, starting at $t$, does not produce an output $y$ at the other end $(B)$ of the linking line before $t+\tau$, or at the input end $A$ (in the form of an incident wave to TDP) before $t+2 \tau$. Thus, if $x$ is Fourier transformed to $X$, its "future" and yet unknown values (those for times larger than $t$ ) can be set to zero, a modification which will not be reflected as an error in the output $y$ obtained from the inverse Fourier transform of $Y$ (calculated from the FDC). The package of $y$, obtained from the truncated inputs $x$, is correct for time domain computations in TDP for the interval from $t$ to $t+2 \tau$.

Thus, the FDC is linked to the FTP by two Fourier transforms, the first being performed with truncated time domain inputs $x$ and the second yielding an output package $y$ valid over a time interval $2 \tau$. The interchange variables $x$ and $y$ are the same as in the conventional EMTP calculations. The only difference is that they are used in packages as inputs and outputs of two Fourier transforms which have to be performed in time intervals of $2 \tau$. At ends $A$ and $B$ of the line, straightforward frequency domain computations are performed to obtain the outputs $Y$ for given inputs $X$.

The application described in the paper is the integration of an external complex system with a study zone. The external system is represented by its frequency domain model. In previous wworks, the external system had to be represented either by fitting of an $R, L, C$ network [2] or by introducing an extended interface $[3,4]$ to reduce the modeling complexity of the external system.

Other applications include the calculation of transients in a system to which an overhead line or a cable is connected, with a simple or complex, frequency dependent termination, as for example a combination of $R, L, C$ elements or a reactor with frequency dependent inductance.

\section{BASIC THEORY}

\subsection{Explanation of the Method}

We refer to Fig.1 to describe the system for which we calculate the electro-magnetic transients. It consists of two parts: the Study Zone and the External System. In conventional EMTP calculations, all computations are performed in the time domain [1,5-7]. Essentially, what is done at any instant $t$ is the following. The incident wave voltage in $A, v_{A, i n c i d}$ is known: it is used to calculate (in addition to the voltage and other variables in $A$ ) the reflected wave voltage $v_{A, \text { reff }}$. Then, with $v_{A, \text { reff }}$ as input to the External System, the time domain computations return a new value $v_{A, \text { incid }}$, valid after a time span $2 \tau$, because of the travel delays involved. The computations proceed step by step, for each $v_{A, \text { incid }}$ and $v_{A, \text { reff }}$, with inmediate response in the Study Zone $A$, and a response to be used later from the External System.

In the proposed hybrid procedure, the computations in the study zone remain the same and are performed using the relevant, existing parts of the EMTP. The difference appears in relation with the External System: the reflected wave voltage $v_{A \text { ref }}$ is not used immediately for time domain processing in the External System. Instead, a set of $v_{A, \text { ref }}$ values is stored, over a time span $2 \tau$; then this $v_{A, \text { reft }}$ package is Fourier transformed into the frequency domain, to yield $V_{A, \text { ref }}$, at a corresponding set of frequencies. Consequently, with $V_{A \text {, reft }}$ as input, frequency domain calculations in the External System will yield a set of $V_{A, \text { incid }}$ 


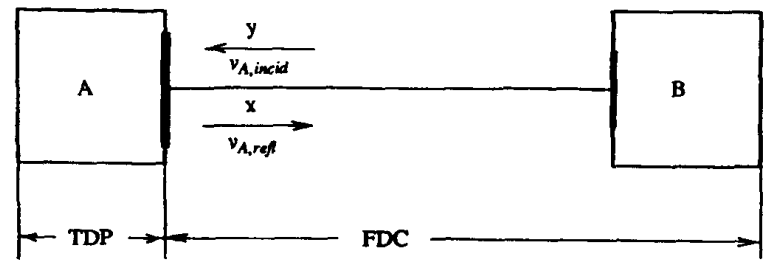

Fig. 1 Basic configuration

TDP - Time Domain Part (Study Zone)

FDC - Frequency Domain Component (External System)

values which, after Fourier transformation, provide a set $v_{A, \text { incid }}$, valid over a time span $2 \tau$ which is shifted with respect to the span for $v_{A, \text { refi }}$ by $2 \tau$ due to the delay inherent in the physical structure of the External System. Thus the information $v_{A \text {, incid }}$ is identical to the one that would have been obtained by time domain procedures, except that it has been obtained in the form of a set over the time span $2 \tau$. Therefore, the FFTs (Fast Fourier Transforms) have to be repeated in intervals of $2 \tau$. Clearly, the longer the linking transmission line, the smaller will be the expenditure for FFTs.

Fig.2 illustrates the ideas underlying the hybrid procedures described above. Time is represented on the horizontal and divided in segments of length $2 \tau$. The Study Zone $A$ has the information processed in the time domain, with vertical arrows indicating how each $v_{A, \text { incid }}$ yields the corresponding $v_{A, \text { reft }}$, for the same time $t$. The double lines at both sides of $A$ indicate FFTs, so that the information outside $A$, in the External System, is processed in the frequency domain. The curved arrow strips indicate the flow of a package and show that, even though the processing is in the frequency domain, the output information appears by a time $2 \tau$ later and thus serves in $A$ as input for the next time period $2 \tau$. In the figure, the curved arrow strips are marked by $B$ in their middle, to reflect the fact that the processes in the External System consist of a reflection in $B$ sandwiched between two travel delays and distortions on the transmission line linking $B$ with the bus of Study Zone $A$.

\subsection{Assumptions for Validity}

The idea of performing part of the calculations in the frequency domain and of using Fourier transforms assumes for validity that the external system is linear and time invariant. Therefore, all nonlinear elements and active switches have to be included in the Study Zone. As already emphasized, the External System must include a transmission line linking it to the Study Zone to provide the travel delay needed for the package calculations of the FFTs. The delay $\tau$ is the value corresponding to light velocity. The actual travel times, for different modes and frequencies, are of course always larger, so that $2 \tau$ is assured to be available for FFT calculations.

There is no need of using modal decomposition in the External Zone and in fact direct phase domain calculations are simpler and more straightforward than the modal alternative. Therefore, the External System should include, if at all possible, all components which are difficult to model in the time domain, for example lines or cables with strongly frequency dependent modal transformations.

\subsection{Computational Steps}

In Fig. 3 the computational process described in the previous section is broken down into the following six steps, marked on the figure. Related computational details will be presented in the next section.

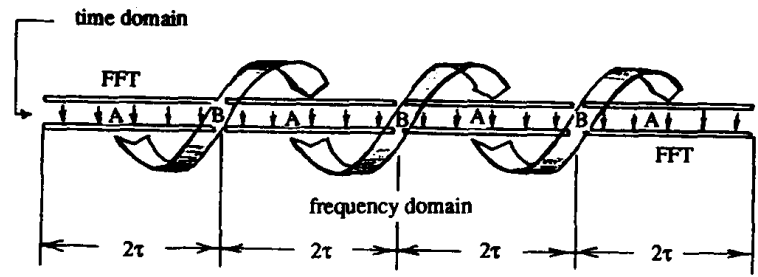

Fig. 2 Illustration of information flow

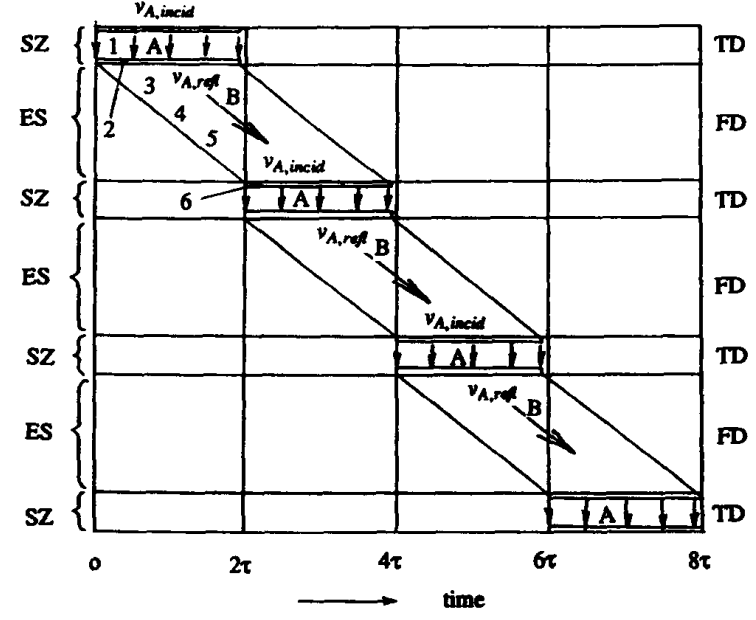

Fig. 3 Algorithmic steps

$$
\begin{aligned}
& \text { SZ - Study Zone } \\
& \text { ES - External System } \\
& \text { TD - Time Domain calculations } \\
& \text { FD - Frequency Domain calculations }
\end{aligned}
$$

1 The incident voltage $v_{A, \text { incid }}$ to the Study Zone (SZ) at $A$ yields via standard EMTP calculations in Time Domain (TD) the reflected voltage $v_{A, \text { reff }}$, calculated at each time step.

2 An FFT is performed on a package of $v_{A, \text { ref }}$ to yield the input $V_{A, r e f}$ in the Frequency Domain (FD) to the line connecting to system $B$ both within the Extemal System (ES).

3 This is the propagation without distortion, in time $\tau$, of the waves corresponding to the package $V_{A, \text { reff }}$ to system $B$; the distortion will be lumped with the transfer function of the next step.

4 At end $B$, the incoming wave package is first "shaped" to take into account the distortion from the previous step; then it is made to interact with system $B$ represented by its driving point admittance $Y_{B}$ so that a reflected wave package is emitted from $B$; finally, it is shaped again to take into account the distortion during its trave back to $A$, indicated in the following step.

5 This is the propagation without distortion of the wave package from $B$ to $A$, in time $\tau$.

6 An FFT converts the incident frequency domain wave package $V_{A, \text { incid }}$ to the time domain package $v_{A, \text { incid }}$.

We note here that the line shaping transfer functions $H_{s h}$ have been lumped with the transfer function $H_{B}$ of system $B$ :

$$
H=H_{s h} H_{B} H_{s h}
$$

This fact has permitted to describe the whole process in the compact block diagram of Fig.4.

\subsection{Computational Details}

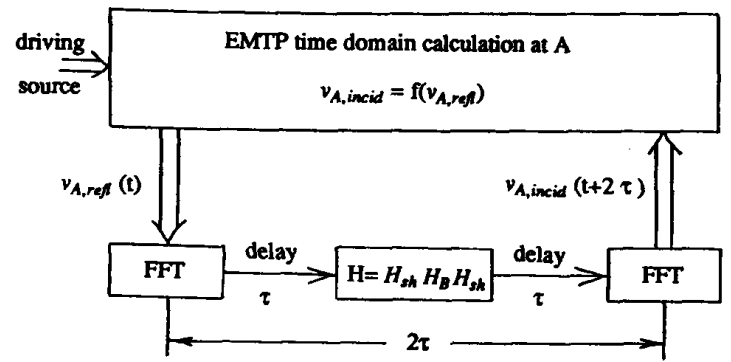

Fig. 4 Block diagram for main computations 


\subsubsection{Backwinding and Shaping}

The frequency domain relation between the voltage $V_{\text {ref }}$ of the wave reflected from the bus at one end of a line and the voltage $V_{\text {incid }}$ of the wave incident at the other end is given in terms of the propagation transfer function $H_{P}$ as

$$
V_{\text {incid }}=H_{P} V_{\text {reft }}
$$

where

$$
H_{P}=e^{-\sqrt{2 X} t}
$$

In the case of a lossless line, Eqn.(3) becomes

$$
H_{0}=e^{-j \omega \tau} U
$$

which corresponds to a pure delay by $\tau$ in the time domain ( $U=$ unity matrix). This suggests to decompose $H_{P}$ into two factors:

$$
H_{P}=H_{0} H_{s h}
$$

where $H_{0}$ represents the delay and $H_{s h}$ represents the shaping effect on the wave due to distortion, i.e. attenuation and frequency dependent propagation velocity. Since in our approach the delay $\tau$ and the shaping effects are taken into account separately (see Fig.4), decomposition (5) is necessary. It is also computationally useful since $H_{s h}$ is, as it will soon become apparent, a much better behaved function in relation to Fourier transforms than $H_{P}$.

From (4) and (5) we obtain

$$
H_{s h}=e^{j \omega r} \times H_{P}
$$

This shows that the shaping function $H_{s h}$ is obtained from the propagation function $H_{P}$ by "backwinding" using $e^{\text {jor }}$; see Fig.5. The many spirals of $H_{P}$ lead in the FFT process to the delay $\tau$ of the output; see Fig.6. Any value calculated over the delay portion $\tau$ of Fig.6b has no informational value as it is a priori known that it must be zero. Fig.6c represents the effect of shaping only on the unit step input of Fig.6a. This is the only information that is in fact needed.

\subsubsection{Accuracy of FFT}

The length $l$ of the line between $A$ and $B$ would normally have a close effect on the selection of the time step $\Delta t$ being used in the EMTP calculations. Let, for example, $l=300 \mathrm{~km}$. Then $\tau=1 \mathrm{~ms}$. We may choose $\Delta r=0.02 \mathrm{~ms}$, so that we will have 100 steps over the span $2 \tau=2$ ms. Unless special attention is given to the selection of the number of steps, it will not be a power of $2\left(2^{7}=128\right.$ in our case), as required by the FFT. Therefore, other past values may have to be chosen outside of the range $2 \tau$ to have a complete set for the FFT $(128-100=28$ values in our case). In fact this approach will prove useful also because it leads to an increased number of frequencies and higher accuracy.

The following remarks can thus be made regarding accuracy: the time step is related to the highest frequency in the spectrum (in our case $1 / 0.02 \mathrm{~ms} \rightarrow 50 \mathrm{kHz}$ ); and the range $T$ of values used is related to the lowest frequency and therefore to the time span for which the calculations are not drifting away (in our case $T=128 \times 0.02 \mathrm{~ms}=2.56 \mathrm{~ms}$ : this is much less than one period of power frequency; the lowest frequency is $1 / 2.56 \mathrm{~ms} \rightarrow 400 \mathrm{~Hz}$ ).

The remarks made above are of tentative nature an indicate only the order of magnitude of the expected values.

\subsubsection{Admittance of External Sustem}

We assume that the system $B$ (which together with the linking line

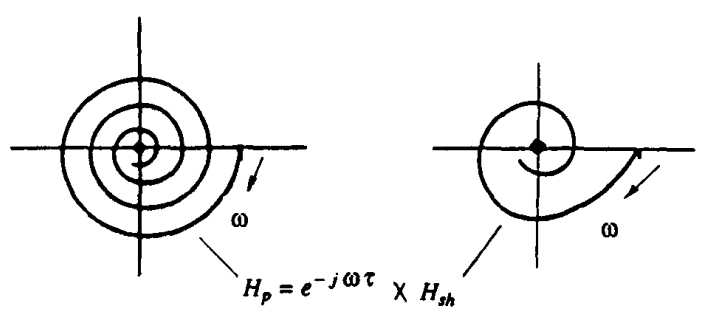

Fig. 5 Pictorial representation of backwinding and shaping (a)

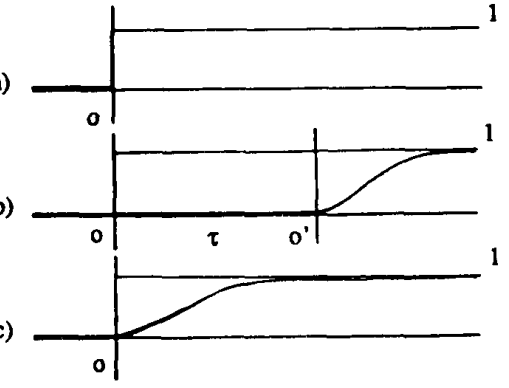

Fig. 6 Unit step responses

(a) Unit step input

(b) Unit step response with $H_{P}$

(c) Unit step response with $H_{s h}$

to $A$ constitutes the External System) consists of a number of buses, in addition to bus $B$; there are transmission lines connected between the buses, and passive elements and generators to the buses; the generators are represented by impedances connected to known voltage sources $E$. Thus we have buses with only impedances connected to them: these are "blind" buses having zero current injections; and buses with known volrages $E$. The computational approach we take will be based on admittances. All calculations refer to the three phases. The corresponding phase domain matrices are not assumed to be balanced: the lines may be untransposed, the (impedance) loads unbalanced. Therefore, whenever functions of matrices appear in the calculations (these are always analytical) they can be trivially evaluated by means of well-known eigenanalysis procedures. form

System $B$ will thus be represented by a matricial equation of the

$$
Y V=I
$$

The first problem is thus the construction of the admittance matrix $Y$ for all frequencies of interest. We note that this process, as well as the subsequent reduction operation, has to be performed only once, in a preprocessing mode, and therefore does not constitute a burden in later calculations.

A transmission line between nodes $i$ and $j$ contributes entries in matrix $Y$ at locations $(i, i),(i, j),(j, i)$, and $(j, j)$, which are equal at each frequency to the elements of its own admittance matrix. These locations have to be interpreted as blocks of $3 \times 3$ elements (for three-phase lines).

Passive elements connected directly to buses will appear as additional admittances in the diagonal of matrix $Y$. Impedances of generators will contribute admittances in four locations, similarly to transmission lines, however, one of the buses is of type $E$.

After the matrix $Y$ has been assembled, Eqn.(7) permits the elimination of all voltages of the blind buses with the result

$$
I_{B}=Y_{B} V_{B}+Y^{\prime} E
$$

If system $B$ has no generators, then Eqn.(8a) reduces to

$$
I_{B}=Y_{B} V_{B}
$$

In the next section we will show that the term $Y^{\prime} E$ in Eqn.(8a) does not affect the transients. Therefore, the presence of generators has to be accounted for, in relation to the transients, only by their contributions to the diagonal elements of matrix $Y$.

2.4.4 Reflection from System $B$

In Eqn.(8a) we can make the following substitutions:

$$
\begin{gathered}
V_{B}=V_{B, \text { incid }}+V_{B, \text { ref }} \\
I_{B}=I_{B \text {,incid }}+I_{B, \text { reff }}=Y_{C}\left(V_{B, \text { incid }}-V_{B, \text { reft }}\right)
\end{gathered}
$$

where $Y_{C}$ is the characteristic admittance (matrix) of the line. This permits us to calculate

$$
V_{B, \text { ref }}=\left(Y_{C}+Y_{B}\right)^{-1}\left(Y_{C}-Y_{B}\right) V_{B, \text { incid }}-\left(Y_{C}+Y_{B}\right)^{-1} Y^{\prime} E
$$

If $E$ would have been zero, we would have obtained 


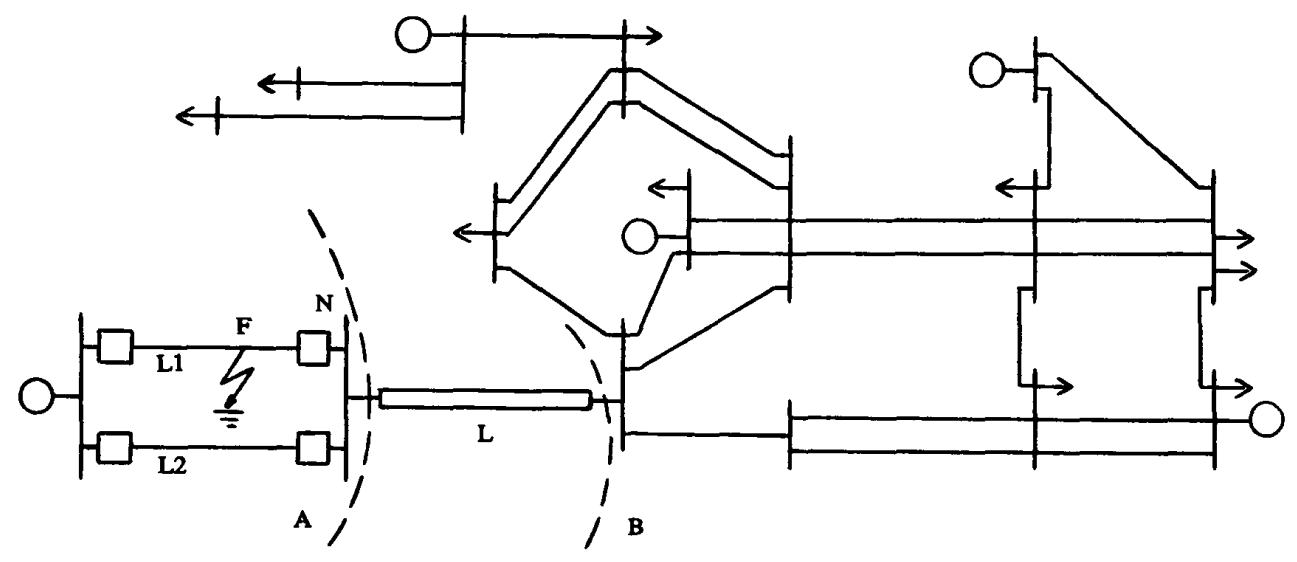

Fig. 7 One-line diagram of System-1

$$
V_{B, \text { reff }}=\left(Y_{C}+Y_{B}\right)^{-1}\left(Y_{C}-Y_{B}\right) V_{B, \text { incid }}
$$

This equation allows us to identify the transfer function

$$
H_{B}=\left(Y_{C}+Y_{B}\right)^{-1}\left(Y_{C}-Y_{B}\right)
$$

which is needed in our calculations (see Eqn.(1) and Fig.4).

Careful examination of Eqn.(10a) permits us to note that the transient depends only on the relation between $V_{B, \text { incid }}$ and $V_{B, \text { ref }}$ and thus the term with $E$ does not contribute to the transient: it affects $V_{B, \text { ref }}$ in a permanent way and consequently it contributes to the (initial) steady state condition. Because of this, we have not included $E$ in our discussions in earlier parts of this paper.

\section{TEST CASES}

The proposed hybrid simulation method has been coded in FORTRAN and interfaced with a special version of the EMTP. In the following, this software package is refered to as HYBRID-EMTP.

Fig. 7 shows the one-line diagram of System-I used to demonstrate the feasibility and accuracy of the HYBRID-EMTP for the simulation of electro-magnetic transients. Subsystem A represents the Study Zone which is composed of a generator connected through $500-\mathrm{kV}$ lines $\mathrm{Ll}$ and $L, 2$ to a large network. Transmission line $L$ is a long line which connects the Study Zone to subsystem B. Line $L$ and subsystem B constitute the External System. Subsystem B comprises $25500-\mathrm{kV}$ transmission lines with 18 right-of-ways. All transmission lines and loads in the system of Fig. 7 are symmetrical. Fig. 8 shows the HYBRID-EMTP simulation of the transients in the system of Fig. 7 corresponding to the following cases.

\subsection{Case-1}

Fig. 8(a) shows the three phase voltages of busbar $\mathrm{N}$ as a result of an L-L-L-G fauit on line $\mathrm{L} 1$ at point $F$. The fault occurs at $t=8 \mathrm{~ms}$ and the faulty line is permanently removed by the breakers, three cycles after the fault occurance. (Each breaker opens at the corresponding current zero-crossing.)

3.2 Case-2

Fig. 8(b) shows the transients of the three phase voltages at busbar $\mathrm{N}$ as a result of simultaneous energization of $\mathrm{L} 1$ and $\mathrm{L} 2$. Initially, all the breakers are open and then simultaneously closed at $t=8 \mathrm{~ms}$.

3.3 Case-3

Fig. 8(c) shows the transients of the three phase voltages at busbar $\mathrm{N}$ as a result of an unsymmetrical L-L-G fault at point $\mathrm{F}$ on line $\mathrm{L} 1$. The fault occurs at $t=8 \mathrm{~ms}$. The faulty line is permanetly removed three cycles after fault inception.

3.4 Case- 4

Fig. 8(d) shows the transients of the three phase voltages at busbar $\mathrm{N}$ as a result of an unsymmetrical L-G fault at point $\mathrm{F}$ on line L1. The fault occurs at $t=8 \mathrm{~ms}$ and is followed by three-pole opening after three cycles.

\subsection{Case-5}

Figs. 9(a) and 9(b) compare the results obtained from the HYBRID-EMTP with those of the EMTP, corresponding to Case-1 and Case-2 respectively. Corresponding to each HYBRID-EMTP simulation study, two simulation studies are conducted by the EMTP. In one study the subsystem B is represented in detail (EXACT-EMTP), and in the other study the Frequency Dependent Network Equivalent (FDNE) program [2] is used to represent subsystem B (FDNE-EMTP).

The comparison (Fig. 9) of the time domain simulation results obtained from HYBRID-EMTP with those obtained from the EXACTEMTP and the FDNE-EMTP verifies the accuracy of the HYBRIDEMTP. Taking the results of the EXACT-EMTP as reference, the maximum deviation of the results from the HYBRID-EMTP and the FDNEEMTP are $6.9 \%$ and $9.3 \%$ for Case- 1 (Fig. 9(a)). The maximum deviations for Case-2 (Fig. 9(b)) are $11.2 \%$ and $7.3 \%$, respectively.

For the reported studies, the simulation time step is $\Delta t=0.002 \mathrm{~ms}$ and the number of samples for the FFT is 1024 . The number of frequency points for the FDNE program is 1000 .

The required CPU-times of a VAX 8600 for the simulation of the electromagnetic transients of System-I (CASE-1) by the EXACT-EMTP, the HYBRID-EMTP and the FDNE-EMTP are 25.73 minutes, 17.89 minutes and 6.63 minutes, respectively. The required preparation time to generate the equivalent of the sub-system B of System-I by the FDNE program is an additional 25.83 minutes. If the length of the line between the study zone and the external system, e.g. line $\mathrm{L}$ in Fig. 7, is small (or there is no line), FDNE is the logical choice and the new methodology is not feasible.

Extensive simulation studies have indicated that the accuracy of the HYBRID-EMTP results can be substantially increased without significant change in the required CPU time. The reason is the logarithmic relationship that exists between the number of samples and the number of multiplications for an FFT procedure. When higher accuracy requires to reduce the time step in the EXACT-EMTP, the CPU time of the EXACT-EMTP increases in proportion. Therefore, compared with the EXACT-EMTP, the required CPU time renders the HYBRID-EMTP increasingly more advantageous as the time step is reduced.

\subsection{Case-6}

Fig. 10 illustrates the single-line diagram of System-II. The Study Zone and line $L$ are identical to those of System-I. Subsystem B consists of six untransposed lines. Therefore, a representation of subsystem B by the FDNE program introduces further approximations while the HYBRID-EMTP can include the unsymmetrical characteristic of subsystem $B$ in the analysis. The system is subjected to an $L-L$ short-circuit at point $F$ on line $\mathrm{L} 1$ at $t=8 \mathrm{~ms}$. Line $\mathrm{L} 1$ is permanently opened three cycles later. Fig. 11 shows the HYBRID-EMTP simulation of the voltage transients at busbar $\mathrm{N}$.

Fig. 12 shows the HYBRID-EMTP, the EXACT-EMTP and the FDNE-EMTP simulation results for one phase at busbar $N$ corresponding to the above mentioned disturbance. The two solid lines in Fig. 12 

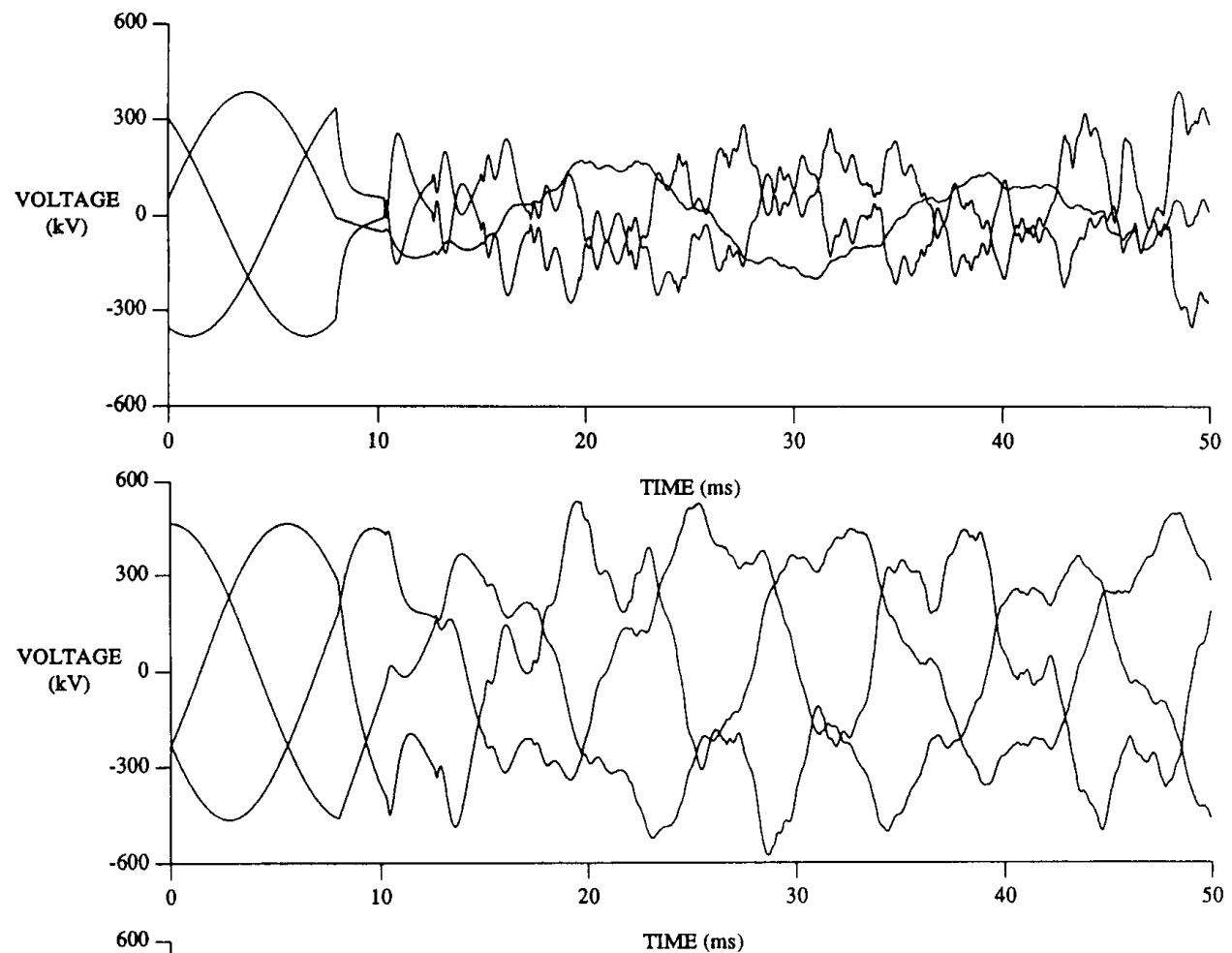

b
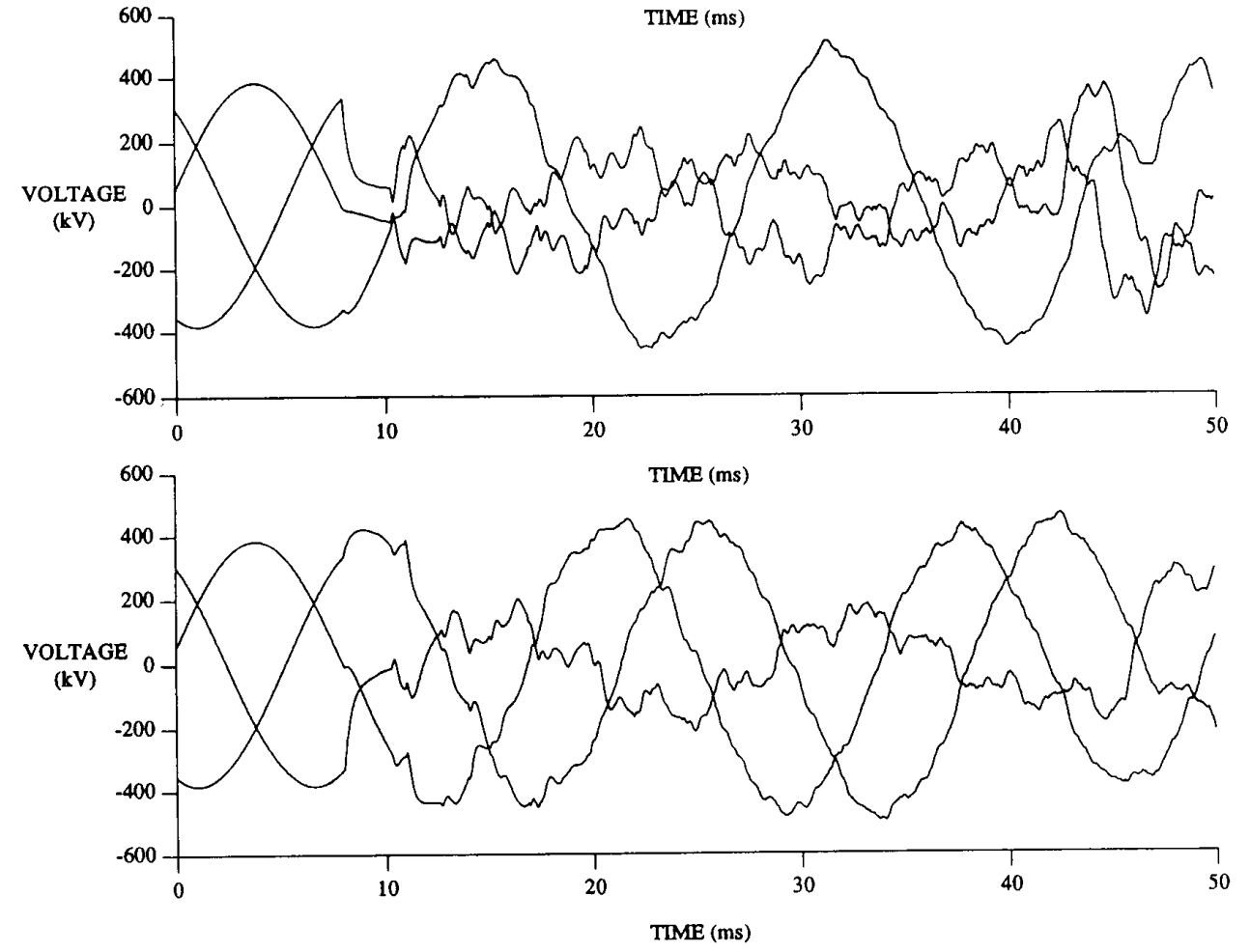

Fig. 8 Three phase voltages at busbar $\mathrm{N}$ of System-I

(a) L-L-L-G fault

(b) Simultaneous enegization of $L 1$ and $L 2$

(c) L-L-G fault

(d) L-G fault 


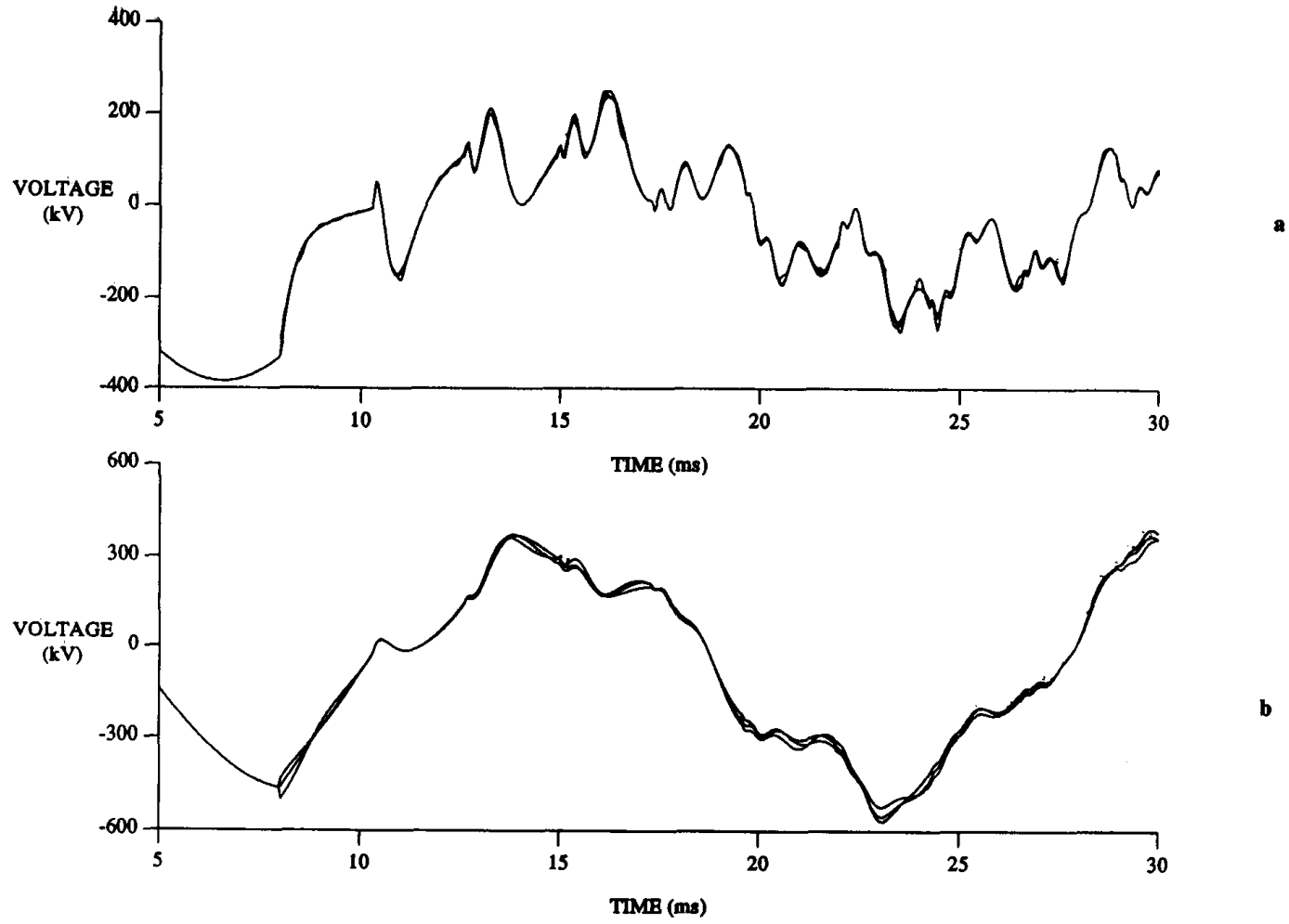

Fig. 9 Comparison of EXACT-EMTP, HYBRID-EMTP and FDNE-EMTP results

(a) Case-1

(b) Case-2

correspond to the EXACT-EMTP and the HYBRID-EMTP results. The broken line corresponds to the FDNE-EMTP simuiatiōn. As can be expected, the results obtained from the FDNE-EMTP do not match closely those from the EXACT-EMTP. Consequently, the hybrid approach is the only satisfactory method for general situations which could include non-symmetrical external systems.

\section{POTENTIAL APPLICATIONS}

Similarly to the EMTP, the HYBRID-EMTP can be used for the analysis of electromagnetic transients in electric power systems with the advantages and properties mentioned in section 3. Anbther potential application of the HYBRID-EMTP is for the analysis of the transients of electric systems which include continuously switching power electronic circuits, e.g. line-commutated HVdc converters, conventional thyristorcontrolled VAR compensators, GTO-thyristor based HVdc converters, GTO-thytistor based VAR compensators, static phaste-shifters, and active filters. In such systems there is a need for vety small time steps to investigate the transient phenomena. Then the required CPU time would prohibit the application of the EMTP to a redlistic size system. However, in the HYBRID-EMTP, the time step can be reduced to the required value without a substantial CPU burden from the external system sirice it is represented in frequency domain.

\section{CONCLUSIONS}

This paper presents an approach for the frequency domain representation of an external system, in the analysis of the electromagnetic transients of a study zone. The study zone is represented in time domain. The requirements are that the external system must be linear, and time invariant and connected to the study zone by a transmission line with travel time $\tau$. In most practical cases, the travel delay $\tau$ can easily be identified in the connection between the two parts of the system. At the connecting bus between the study zone and the external system, Fast Fourier Transforms (FFTs) provide the conversion from time domain to frequency domain and vice versa. The travel delay $\tau$ of the transmission line provides the possibility for the FFTs to be carried out only at time intervals of $2 \tau$. Thus, the approach is computationally efficient, especially in the case of large external systems.

Other important advantages of the method are the following.

The accuracy and the simplicity of the frequency domain modeling is preserved, sitce no approximation or fitting is required. Thus, the resulting accuracy cannot be matched by equivalencing thethods. It can in fact exceed that of the EMTP, without loss of computational efficiency

Complex frequency dependence in the external system does not lead to any difficulty or limitations since the representation is directly in the frequency domain. Consequently, transmission lines can be modeled directly in the phase domain and modal decomposition is neither necessary nor

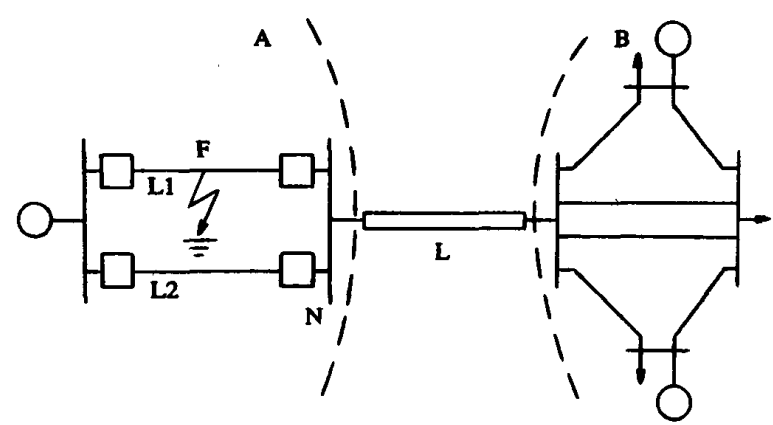

Fig. 10 One-line diagram of System-II 


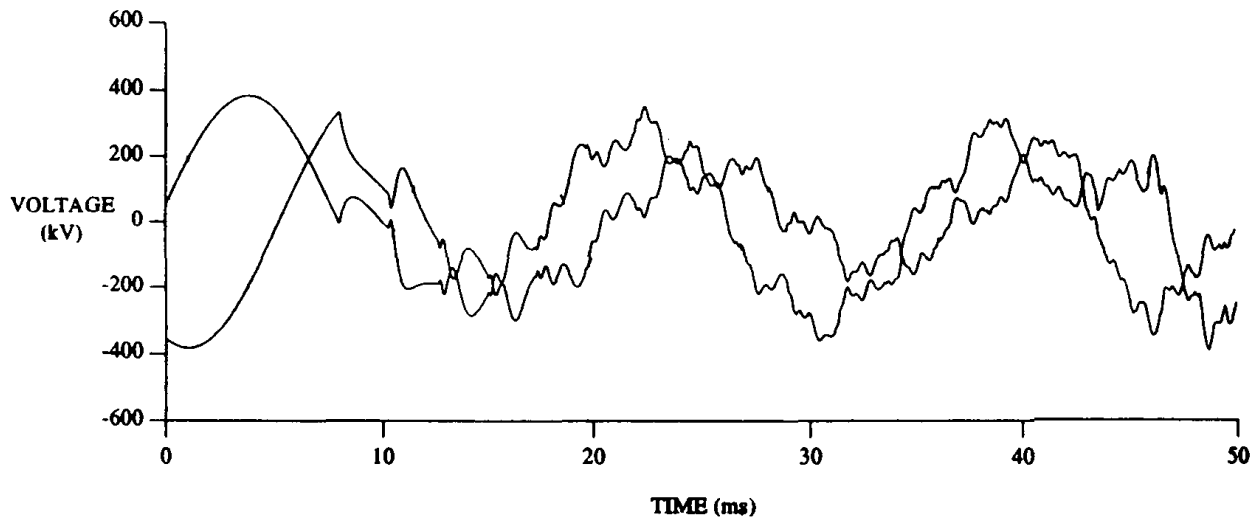

Fig. 11 Voltage transients corresponding to the two phases subjected to L-L short circuit

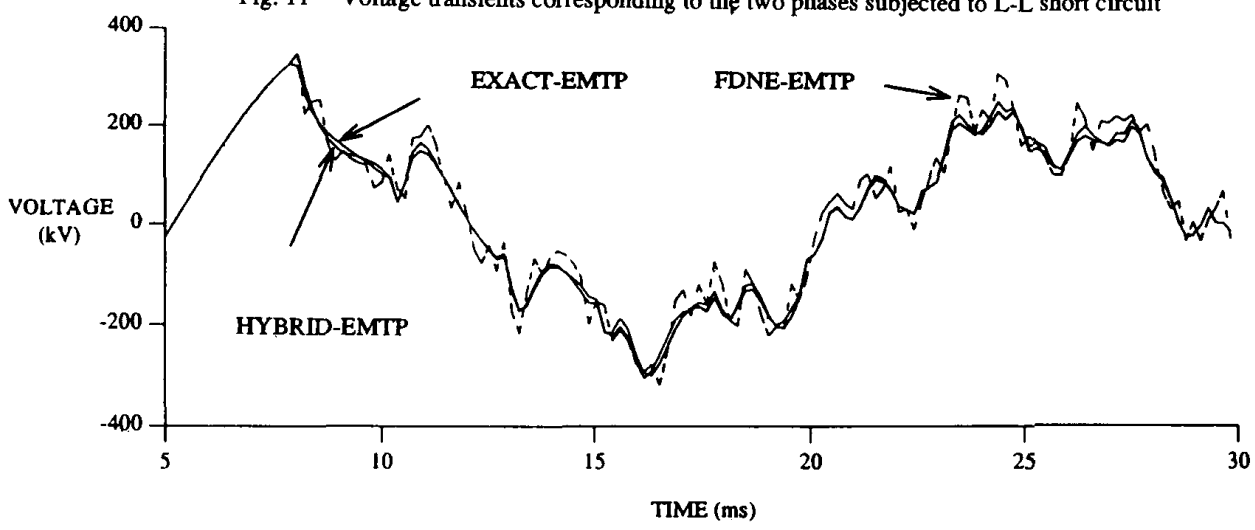

Fig. 12 Comparison of EXACT-EMTP, HYBRID-EMTP and FDNE-EMTP results (Case-6)

useful. Lines and components can have any degree of asymmetry a condition which, beside the proposed hybrid approach, can at present be simulated only by the exact representation of the complete system with the EMTP.

Even components for which only frequency domain information exists, but no appropriate EMTP nodel, can be represented with full accuracy as (part of) the external system. For example, a three-phase reactor can be directly included in the model by using its frequency dependent admintapce matrix from terminal measurements.

The proposed hybrid approach is robust since it does not require any fitting procedures that may have problems of convergence and accuracy and are available only for the individual modes into which symmetrical systems can be decomposed.

The computational feasibility of the proposed hybrid method is demonstrated on two test systems. The electro-magnetic transients of the test systems have been aiso investigated by the EMTP. Comparison of the results obtained from the hybrid method with those obtained from the EMTP verifies the computational feasibility and the accuracy of the proposed hybrid simulation method.

\section{ACKNOWLEDGEMENT}

Financial support from the Engineering and Natural Sciences Research Council of Canada is gratefully acknowledged.

\section{REFERENCES}

[1] H.W. Dommel, "Digital Computer Solution of Electromagnetic Transients in Single- and Multi-Phase Networks", IEEE Trans., Vol. PAS-88, pp. 388-399, April 1969.

[2] A.S. Morched and V. Brandwajn, "Transmission Network Equivalents For Electromagnetic Transient Studies", IEEE Trans.,
Vol. PAS-102, pp. 2984-2994, September 1983.

[3] J. Reeve and R. Adapa, "A New Approach to Dynamic Analysis of AC Network Incorporating Detailed Modeling of DC Systems. Part I: Principles and Implementations", IEEE Trans., Vol. PWRD-3, No. 4, pp. 2005-2011, October 1988.

[4] R. Adapa and J. Reeve, "A New Approach to Dynamic Analysis of AC Network Incorporating Detailed Modeling of DC Systems. Part II: Application to Interaction of DC and Weak AC Systems", IEEE Trans., Vol. PWRD-3, No. 4, pp. 2012-2019, October 1988.

[5] H.W. Dommel and S.W. Meyer, "Computations of Electromag netic Transients", Proceedings of the IEEE, Vol. 62, No. 7, pp 983-993, 1974.

[6] H.W. Dommel, "Nonlinear and Time-Varing Elements in Digita Simulation of Electromagnetic Transients", IEEE Trans., Vol PAS-90, pp. 2561-2567, Nov./Dec. 1971.

[7] G. Gross and M.C. Hall, "Synchronous Machine and Torsional Dynamics in Computation of Electromagnetic Transients", IEEE Trans., Vol. PAS-97, pp. 1074-1086, 1978.

Adam Semlyen (F87) was born and educated in Rumania where he obtained a Dipl. Ing. degree and his Ph.D. He started his career with an electric power utility and held an academic position at the Polytechnic Institute of Timisoara, Rumania. In 1969, he joined the University of Toronto, where he is a prafessor in the Department of Electrical Engineering. His research interests include steady state and dynamic analysis of power systems, electromagnetic transients, and power system optimization.

Reza Iravani (M'85) received his B.Sc. degree in electrical engineering in 1976 from Tebran Polytechnique University and started his career as a consulting engineer. He received his M.Sc. and $\mathrm{Ph} . \mathrm{D}$. degrees in electrical engineering from the University of Manitoba, Canada in 1981 and 1985 respectively. Presently, he is an associate professor in the Department of Electrical Engineering at the University of Toronto. His research interest include power system dynamics, control, and simulation. 